\title{
Utilisation Des Tubes A Diffusion Passive Pour La Surveillance De La Pollution Automobile Dans La Ville De Meknes
}

\author{
Ibrahim El Ghazi, PhD \\ Imane Berni, PhD \\ Aziza Menouni, PhD
}

Université Moulay Ismail, Faculté des Sciences de Meknès, Maroc

Prof. Marie-Paule Kestemont

Université Catholique de Louvain, Louvain-la-Neuve, Belgique

Prof. Mohammed Amane

Prof. Samir El Jaafari

Université Moulay Ismail, Faculté des Sciences de Meknès, Maroc

Doi:10.19044/esj.2018.v14n23p19 URL:http://dx.doi.org/10.19044/esj.2018.v14n23p19

\begin{abstract}
Road traffic emission is one of the major sources of air pollution which can cause several human health problems including cardiorespiratory diseases. The aim of our study is to monitor air quality in Meknes city (Morocco) by measuring nitrogen dioxide $\left(\mathrm{NO}_{2}\right)$ and benzene $\left(\mathrm{C}_{6} \mathrm{H}_{6}\right)$ concentration mainly generated by road traffic.

To this end, we deployed passive diffusion tubes at 14 sampling sites during two measurement campaigns in the summer of 2014 and the winter of 2015 using car and underground proximity sites. In parallel with the winter measurement campaign, road traffic counting sessions were conducted on the main roads of the city in order to determine average daily traffic intensity.

Results of this study show that the atmospheric concentrations of $\mathrm{NO}_{2}$ and $\mathrm{C}_{6} \mathrm{H}_{6}$ reach maximum values in the city center and decrease towards its periphery. The average value of $\mathrm{NO}_{2}$ in all targeted sites was around 32, $59 \mu \mathrm{g}$ $/ \mathrm{m}^{3}$, which is lower than the EU limit of $40 \mu \mathrm{g} / \mathrm{m}^{3}$. The average concentration of $\mathrm{C}_{6} \mathrm{H}_{6}$ in Meknes was equal to $1,77 \mu \mathrm{g} / \mathrm{m}^{3}$, a value close to the quality objective set by the European Union $\left(2 \mu \mathrm{g} / \mathrm{m}^{3}\right)$ and well below the annual Moroccan limit $\left(10 \mu \mathrm{g} / \mathrm{m}^{3}\right)$.

The use of GIS (geographic information system) for coupling the results of measurement campaigns and those of traffic counting made it possible to determine the areas most affected by these tracers and thus to set up very high spatial resolution cartography.
\end{abstract}


Keywords: Passive diffusion tubes, Air quality, Nitrogen Dioxide, Benzene,

\section{Résumé}

Dans cette étude nous nous sommes intéressés à la surveillance de la qualité de l'air dans la ville de Meknès en mesurant les teneurs du dioxyde d'azote $\left(\mathrm{NO}_{2}\right)$ et du benzène $\left(\mathrm{C}_{6} \mathrm{H}_{6}\right)$ générés par le trafic routier. Nous avons ainsi mené deux campagnes de mesures, durant l'été 2014 et l'hiver 2015 en utilisant des sites de proximité automobile et de fond.

Pour ce faire, des tubes à diffusion passive ont été déployés dans 14 sites de prélèvements. En parallèle avec la campagne de mesure hivernale des deux traceurs $\left(\mathrm{NO}_{2}\right.$ et $\left.\mathrm{C}_{6} \mathrm{H}_{6}\right)$, des sessions de comptage du trafic routier ont été réalisées dans les principaux axes routiers de la ville.

Les résultats de ce travail montrent que les concentrations atmosphériques du $\mathrm{NO}_{2}$ et du $\mathrm{C}_{6} \mathrm{H}_{6}$ atteignent des valeurs maximales dans le centre-ville et décroissent vers sa périphérie.

La teneur moyenne du $\mathrm{NO}_{2}$ dans tous les sites étudiés est, d'environ 32,59 $\mu \mathrm{g} / \mathrm{m}^{3}$, valeur inférieure à la limite fixée par l'Union Européenne $\left(40 \mu \mathrm{g} / \mathrm{m}^{3}\right)$. La concentration moyenne du $\mathrm{C}_{6} \mathrm{H}_{6}$ à Meknès est égale à $1,77 \mu \mathrm{g} / \mathrm{m}^{3}$, valeur proche de l'objectif de qualité fixé par l'Union Européenne $\left(2 \mu \mathrm{g} / \mathrm{m}^{3}\right)$ et bien inférieure à la limite annuelle marocaine $\left(10 \mu \mathrm{g} / \mathrm{m}^{3}\right)$.

Le couplage des résultats des campagnes de mesures et ceux du comptage sous SIG (système d'information géographique) a permis de déterminer les zones les plus touchées par ces traceurs et de réaliser ainsi une cartographie à haute échelle de résolution spatiale du $\mathrm{NO}_{2}$ et du $\mathrm{C}_{6} \mathrm{H}_{6}$.

Mots clés: Tubes à diffusion passive, qualité de l'air, dioxyde d'azote, benzène

\section{Introduction:}

La pollution de l'air a des répercussions sur 1'Homme, la faune et la flore. Selon l'OMS, 7 millions de décès prématurés sont attribués chaque année à la pollution atmosphérique dans les zones urbaines et rurales (OMS, 2018). Les pluies acides, la perturbation des écosystèmes, l'eutrophisation et la dégradation du patrimoine et du cadre de vie sont les principales conséquences environnementales de la détérioration de la qualité de l'air. Le monitoring et la surveillance des teneurs des polluants atmosphériques sont d'une importance cruciale et peuvent être réalisés par des outils de mesures passifs (tubes à diffusion passive, radiellos et badges) et actifs (stations télémétriques fixes et laboratoires mobiles). Ces deux derniers sont capables de quantifier les teneurs des polluants de l'air en temps réel et en continu (résolution temporelle très élevée) alors que les tubes à diffusion passive 
peuvent être utilisés pour étudier la pollution de l'air au milieu intérieur (Tahir et al ., 2015 ; Zabiegała et al., 2007) comme à l'extérieur (Hůnová, 2017) et ils permettent de réaliser des cartographies à haute échelle de résolution spatiale (Freeman et al., 2017 ; Mine Evci et al., 2017).

$\mathrm{Au}$ Maroc, l'observation de la géolocalisation des stations de mesure montre que 7 régions administratives parmi les 16 existantes (anciennes subdivisons administratives) ne sont pas dotées de stations de surveillance de la qualité de l'air. Parmi ces régions, figure l'ex-région de Meknès-Tafilalet dont la superficie dépasse $79210 \mathrm{~km}^{2}$ pour une population totale de plus de 2 millions d'habitants. Au niveau de cette région, la pollution atmosphérique est localisée à Meknès et ses abords immédiats. Les statistiques montrent que dans cette zone se concentre environ $89 \%$ du parc automobile de la région et la plus grande partie des industries polluantes. Le trafic automobile rejette annuellement dans l'atmosphère $1956 \mathrm{t}$ de $\mathrm{SO}_{2}, 20454 \mathrm{t}$ du NOx, $780 \mathrm{t}$ de matière en suspension et $13 \mathrm{t}$ de $\mathrm{Pb}$. La Ville de Meknès est responsable à elle seule de la production de 60\% de ces émissions (MRE, 2001). Ces statistiques ont été actualisées, complétées et appuyées par de nouvelles recherches (Ait Bouh et al., 2013 ; Boularab et al., 2015 ; El Ghazi et al., 2015).

En absence d'une station télémétrique au niveau du chef-lieu de la région, l'utilisation des tubes à diffusion passive pour le monitoring et la cartographie de la qualité de l'air s'avère pertinente.

L’objectif de notre étude est double :

- déterminer la part du secteur du transport dans la pollution de l'air au niveau de la Ville de Meknès à travers la surveillance et le monitoring du $\mathrm{NO}_{2}$ et du $\mathrm{C}_{6} \mathrm{H}_{6}$ (traceurs de la pollution automobile);

- réaliser une cartographie à haute échelle de résolution spatiale des teneurs du $\mathrm{NO}_{2}$ et du $\mathrm{C}_{6} \mathrm{H}_{6}$ dans l'air ambiant au niveau de la Ville de Meknès.

\section{Matériels et Méthodes : \\ Présentation de la zone d'étude}

La surveillance des variations spatio-temporelles des teneurs du $\mathrm{NO}_{2}$ et du $\mathrm{C}_{6} \mathrm{H}_{6}$ a été réalisée dans la ville de Meknès $\left(33^{\circ} 53^{\circ}\right.$ Nord, $5^{\circ} 33^{\circ}$ Ouest), située au nord-ouest du Maroc à $564 \mathrm{~m}$ d'altitude (fig.1). Le climat est de type méditerranéen, caractérisé par un hiver humide et froid et un été sec et chaud. Vue sa position géographique, la ville représente un carrefour entre les plaines atlantiques, les collines pré-rifaines, le Moyen-Atlas et les hauts plateaux de l'Oriental. Pour une population d'environ 632079 habitants, Meknès est classée parmi les six grandes villes du Royaume (Sai et al., 2014). Cette ville est soumise à une pollution atmosphérique issue du trafic routier, des zones d'activités industrielles et du secteur d'artisanat (Abdouh et al., 2004 ; MRE, 2001). 


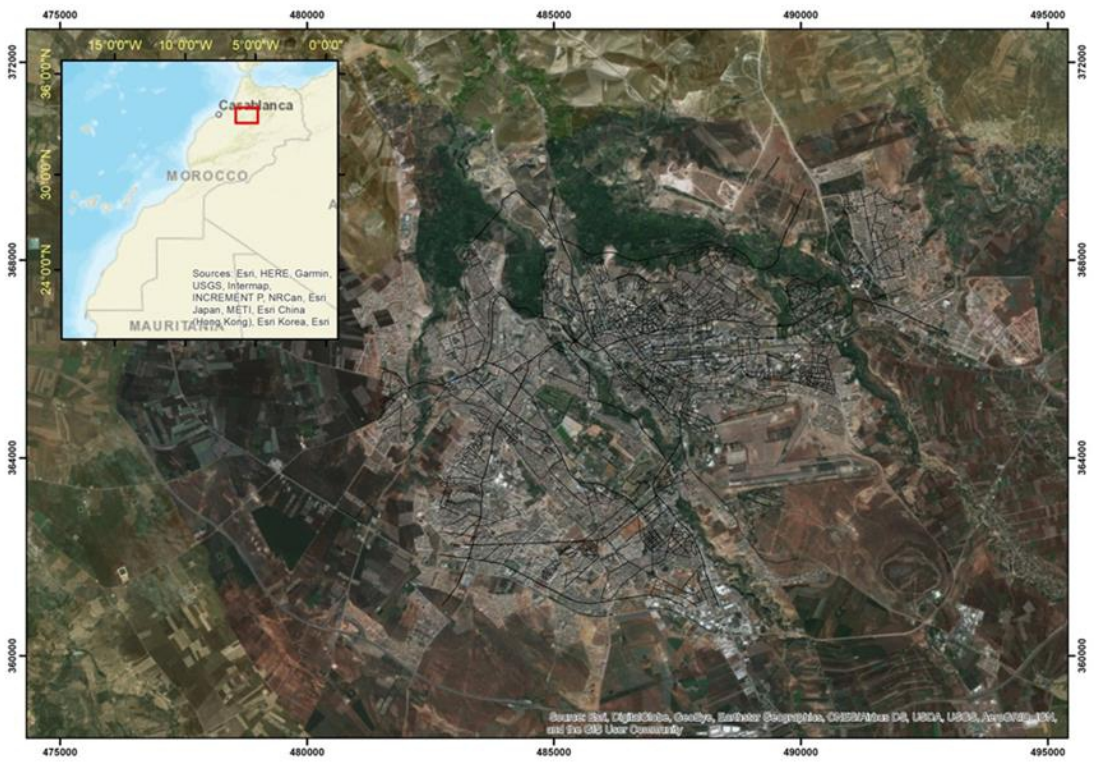

Fig.1. Géolocalisation de la ville de Meknès (El Ghazi et al., 2015)

\section{Echantillonnage de $\mathrm{NO}_{2}$}

Pour le monitoring des teneurs de $\mathrm{NO}_{2}$ des tubes à diffusion passive, comportant trois grilles en acier inoxydable imprégnées du Triéthanolamine, (Atkins, 1990 ; NBN EN 16339) ont été déployés au niveau de 14 sites de prélèvements. Le choix de l'échantillonnage passif repose sur la facilité de sa mise en œuvre, le fonctionnement sans raccordement électrique ou d'utilisation de pompe et la possibilité de couvrir une large zone géographique avec un grand nombre d'échantillonneurs à faible coût. Les préleveurs utilisés consistent en tubes acrylique de $74 \mathrm{~mm}$ de long et de $9,5 \mathrm{~mm}$ de diamètre. Les échantillonneurs ont été fixés verticalement à des supports droits, à 3-4 m audessus du niveau du sol. Un abri spécifique était utilisé pour la protection du matériel de prélèvement contre les intempéries et l'influence du vent (Plaisance et al., 2004). Après deux semaines d'exposition, les tubes palmes sont relevés et le $\mathrm{NO}_{2}$ piégé dans les grilles est extrait via l'ajout de $3 \mathrm{ml}$ du réactif de Saltzman (Atkins, 1990 ; NBN EN 16339) et sa quantité dans les extraits est ensuite dosée par UV pour une longueur d'onde de $542 \mathrm{~nm}$.

\section{Echantillonnage du $\mathrm{C}_{6} \mathrm{H}_{6}$}

Pour le $\mathrm{C}_{6} \mathrm{H}_{6}$, les tubes utilisés sont du type Perkin Elmer. Ils ont une longueur de $90 \mathrm{~mm}$, un diamètre extérieur de $6,35 \mathrm{~mm}$ et un diamètre intérieur de $5 \mathrm{~mm}$. Chaque tube porte un numéro d'identification unique gravé. Ils sont fermés hermétiquement, avant et après les prélèvements par des bouchons en laiton type Swagelok ${ }^{\circledR}$, comprenant des férules en téflon. La durée d'exposition de ces tubes est de 30 jours. Le prélèvement et l'analyse sont 
réalisés selon la norme ISO 16017-2 : 2003. Après échantillonnage, les cartouches sont analysées par désorption thermique suivie d'une chromatographie en phase gazeuse avec comme détecteur un spectromètre de masse.

\section{Géolocalisation des sites de prélèvements}

Les capteurs passifs ont été déployés dans 14 sites de mesure répartis en sites de proximité automobiles (P) et ceux de fond (F) (fig.2 et tableau 1).

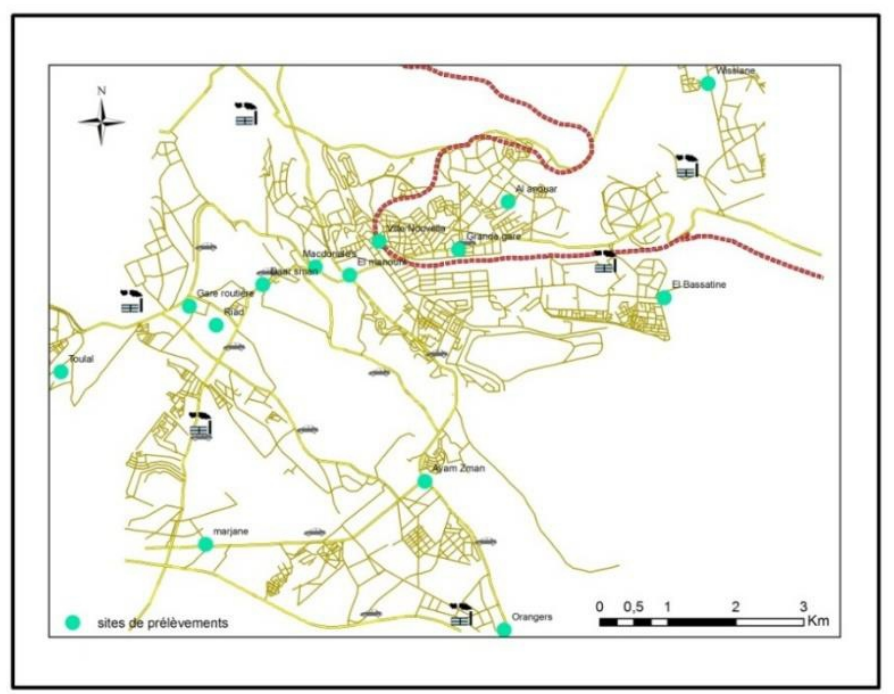

Fig.2. Géolocalisation des sites de prélèvements

Tableau 1 : Répartition des sites de prélèvements par type et par localisation

\begin{tabular}{|c|c|c|}
\hline points & Lieu & Typologie \\
\hline P1 & point d'intersection entre la route nationale n ${ }^{\circ}$ 13 et la route nationale ${ }^{\circ} 6$ & trafic \\
\hline P2 & point d'intersection de l'avenue Bir Anzarane et du boulevard Zitoune & trafic \\
\hline P3 & avenue zitoune (district de Marjane) & trafic \\
\hline P4 & point d'intersection de l'avenue Bir Anzarane et l'avenue des forces armées royales & trafic \\
\hline P5 & avenue des forces armées royales près de la grande gare & trafic \\
\hline P6 & point d'intersection de l'avenue Mohamed VI et l'avenue des forces armées royales & trafic \\
\hline P7 & rue Daar smane, point de jointure de l'ancienne Medina à la ville nouvelle & trafic \\
\hline P8 & La gare routière de la ville de Meknès & trafic \\
\hline F1 & Toulal & Fond \\
\hline F2 & Riad & Fond \\
\hline F3 & ville nouvelle près la gare d'el Amire Abdelkader & Fond \\
\hline F4 & Hacienda & Fond \\
\hline F5 & El Bassatine & Fond \\
\hline F6 & Wisslane & Fond \\
\hline
\end{tabular}




\section{Comptage du trafic routier}

En parallèle avec la campagne de mesure hivernale de $\mathrm{NO}_{2}$ et de $\mathrm{C}_{6} \mathrm{H}_{6}$, des sessions de comptage du trafic routier ont été réalisées dans les principaux axes routiers de la ville. Le comptage a été effectué par des opérateurs munis de cliquers manuels. Pour étudier les variations inter-journalières et les fluctuations du trafic routier au cours des journées ouvrables et fermées, une semaine de comptage par site était nécessaire. 12 sites de comptages ont été sélectionnés (fig.3) et le choix de leurs emplacements a été dicté par la nature $\mathrm{du}$ tronçon routier et la géolocalisation des échantillonneurs passifs. Les comptages ont été réalisés sur des laps de temps de 15 minutes.

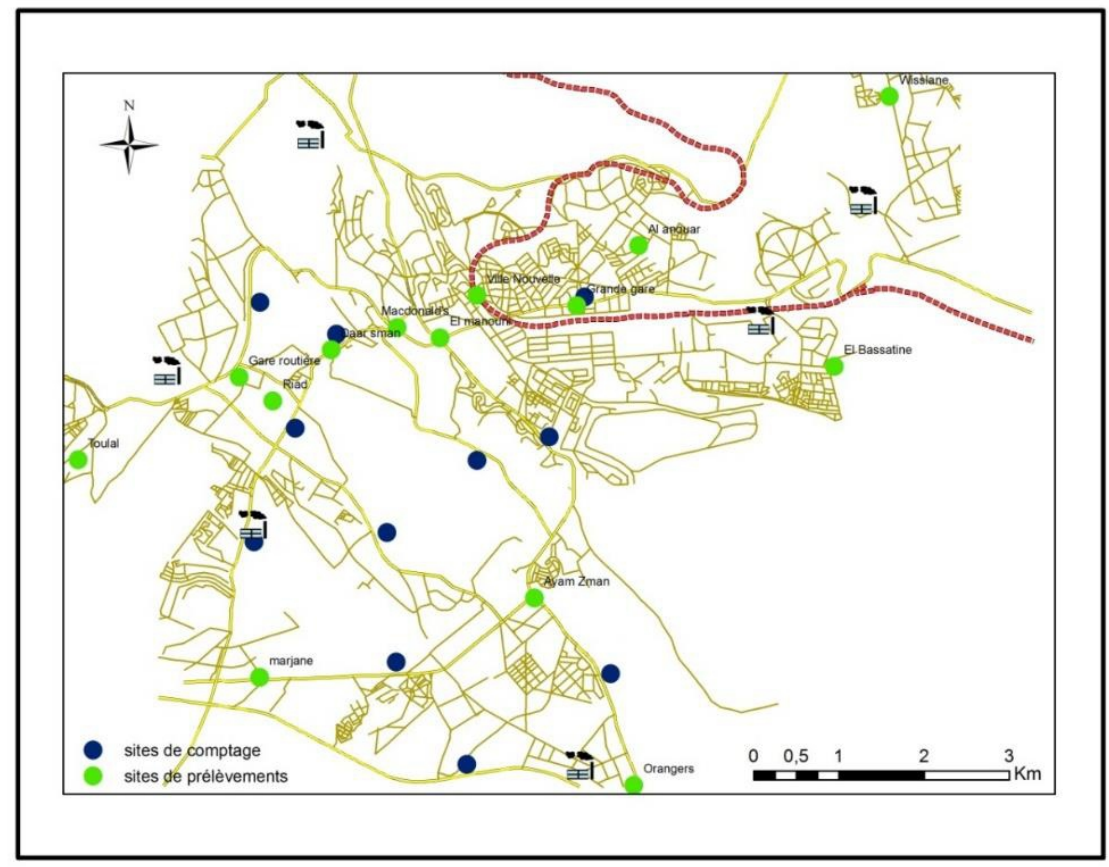

Fig.3. Géolocalisation des points de comptage

\section{Cartographie des teneurs de $\mathrm{NO}_{2}$ et de $\mathrm{C}_{6} \mathrm{H}_{6}$}

La cartographie des concentrations de $\mathrm{NO}_{2}$ et de $\mathrm{C}_{6} \mathrm{H}_{6}$ a été réalisée par interpolation spatiale selon la méthode de pondération de type Krigeage. Cette technique permet l'estimation des variables continues dans l'espace à des endroits inconnus à partir des valeurs mesurées dans des emplacements déterminés. 


\section{Résultats et discussions : \\ Etude des teneurs du $\mathrm{NO}_{2}$}

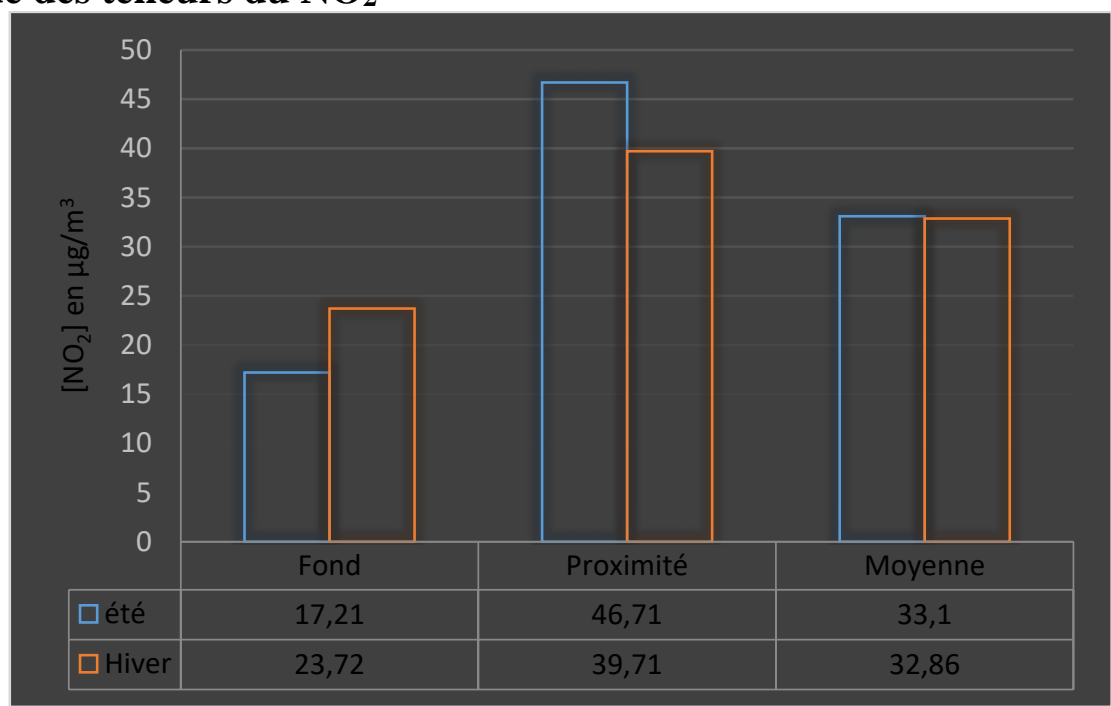

Figure 4. Concentrations moyennes du $\mathrm{NO}_{2}$ dans la ville de Meknès durant la période d'étude

Les plus fortes concentrations du $\mathrm{NO}_{2}$ détectées, ont été mesurées au niveau des sites de proximité automobiles $\left(41,68 \mu \mathrm{g} / \mathrm{m}^{3}\right)$. Par contre, les plus faibles teneurs ont été enregistrées dans les sites de fond $\left(20,47 \mu \mathrm{g} / \mathrm{m}^{3}\right)$ (fig.4). La concentration moyenne du $\mathrm{NO}_{2}$ mesurée durant la campagne estivale $\left(33,10 \mu \mathrm{g} / \mathrm{m}^{3}\right)$ est très proche de celle notifiée en hiver $\left(32,86 \mu \mathrm{g} / \mathrm{m}^{3}\right)$ (fig.4).

En hiver, la concentration moyenne du $\mathrm{NO}_{2}$ notée au niveau des sites de proximité automobiles est égale à $39,71 \mu \mathrm{g} / \mathrm{m}^{3}$ alors que celle des sites de fond est de $23,72 \mu \mathrm{g} / \mathrm{m}^{3}$.

En été, la concentration moyenne du $\mathrm{NO}_{2}$ était de $46,71 \mu \mathrm{g} / \mathrm{m}^{3}$ au niveau des sites de proximités automobiles et de $17,21 \mu \mathrm{g} / \mathrm{m}^{3}$ dans les sites de fond (fig.4).

Le taux des précipitations au cours de la campagne hivernale a conduit, probablement, à la diminution des teneurs de $\mathrm{NO}_{2}$ dans l'air, vu sa solubilité dans l'eau qui induit sa décomposition en acide nitreux et nitrique (Meybeck et al., 2000).

Tous les sites de fond présentent des teneurs de $\mathrm{NO}_{2}$ inférieures à 40 $\mu \mathrm{g} / \mathrm{m}^{3}$, seuil limite admissible en Europe (Directive 2008/50/CE). Cinq sites de proximité automobile sur 8 présentent des teneurs dépassant $40 \mu \mathrm{g} / \mathrm{m}^{3}$. Parmi ces sites, deux dépassent la valeur limite marocaine de $50 \mu \mathrm{g} / \mathrm{m}^{3}$ (figure $5)$. 


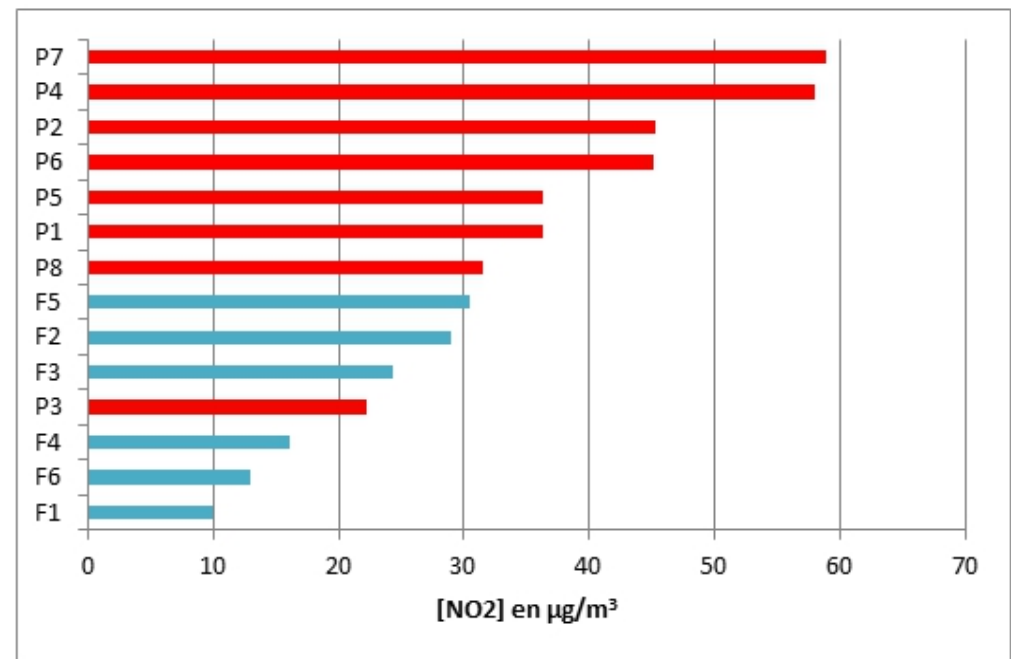

Fig.5. Distribution des sites par concentration de $\mathrm{NO}_{2}$ (résultats des campagnes de mesure)

\section{Cartographie des teneurs de $\mathrm{NO}_{2}$}

Les concentrations atmosphériques de $\mathrm{NO}_{2}$ atteignent des valeurs maximales dans le centre-ville et tendent à décroitre vers sa périphérie (fig.6(a) et 6(b)).

Les plus fortes concentrations relevées correspondent à des sites de proximité automobile du centre-ville : P7 situé au niveau de la rue dar smane $\left(58,37 \mu \mathrm{g} / \mathrm{m}^{3}\right)$, P4 qui représente l'intersection entre l'avenue des forces armées royales et l'avenue Bir Anzarane $\left(57,96 \mu \mathrm{g} / \mathrm{m}^{3}\right)$, P2 point d'intersection de l'avenue Bir Anzarane et le boulevard zitoune $\left(48,23 \mu \mathrm{g} / \mathrm{m}^{3}\right)$ et P6 croisement de l'avenue Mohamed VI et des Forces armées Royales $\left(45,12 \mu \mathrm{g} / \mathrm{m}^{3}\right)$.

En effet, ces axes routiers sont caractérisés par un trafic quotidien important, qui est à l'origine d'une grande partie des émissions de $\mathrm{NO}_{2}$ (Meybeck et al., 2000 ; Laurinavičienè et al., 2013).

Les fortes teneurs signalées au niveau du site P7, localisé au niveau de la rue dar smane, emprunté par pas moins de 12000 véh/j dépassent ceux mesurées au niveau du site $\mathrm{P} 4$, point d'intersection traversé par plus de 24000 véh/j. Ceci peut s'expliquer par la structure de la rue non-propice pour une meilleure dispersion de $\mathrm{NO}_{2}$ et favorise par contre son accumulation. Le rapport hauteur/largeur au niveau de cette rue est supérieur à 1 ce qui permet de la qualifier de rue canyon (Chelala et al., 2007).

Les sites présentant des concentrations inférieures à $15 \mu \mathrm{g} / \mathrm{m}^{3}$ sont ceux de Wisslane (F6) et Toulal (F1) vu leurs géolocalisation.

Pour les sites F6 et P1, malgré leurs proximités de la cimenterie de Lafarge et du quartier industriel de sidi Bouzekri, nous avons constaté que les 
teneurs mesurées sont inférieures à la valeur limite admissible. Ces résultats concordent avec ceux des enquêtes réalisées par le Ministère de l'Environnement marocain, qui ont montré que le trafic routier est responsable de $75 \%$ des émissions de $\mathrm{NO}_{2}$ et celles du secteur industriel n'excède pas 25 $\%$ (MRE, 2001). Ceci rime, également, avec l'étude réalisée au niveau de la ville de Toulouse qui a confirmé que les plus fortes teneurs de $\mathrm{NO}_{2}$ ont été décelées dans les sites de proximité automobiles du centre-ville (Meybeck et al., 2000). De même, une autre étude réalisée dans la ville de Kaunas (deuxième grande ville de Lituanie) a montré que les émissions de $\mathrm{NO}_{2}$ sont fortement liées à la densité du trafic routier et que les plus fortes concentrations de $\mathrm{NO}_{2}$ ont été enregistrées au niveau des stations de proximité automobiles (Laurinavičienè et al., 2013).

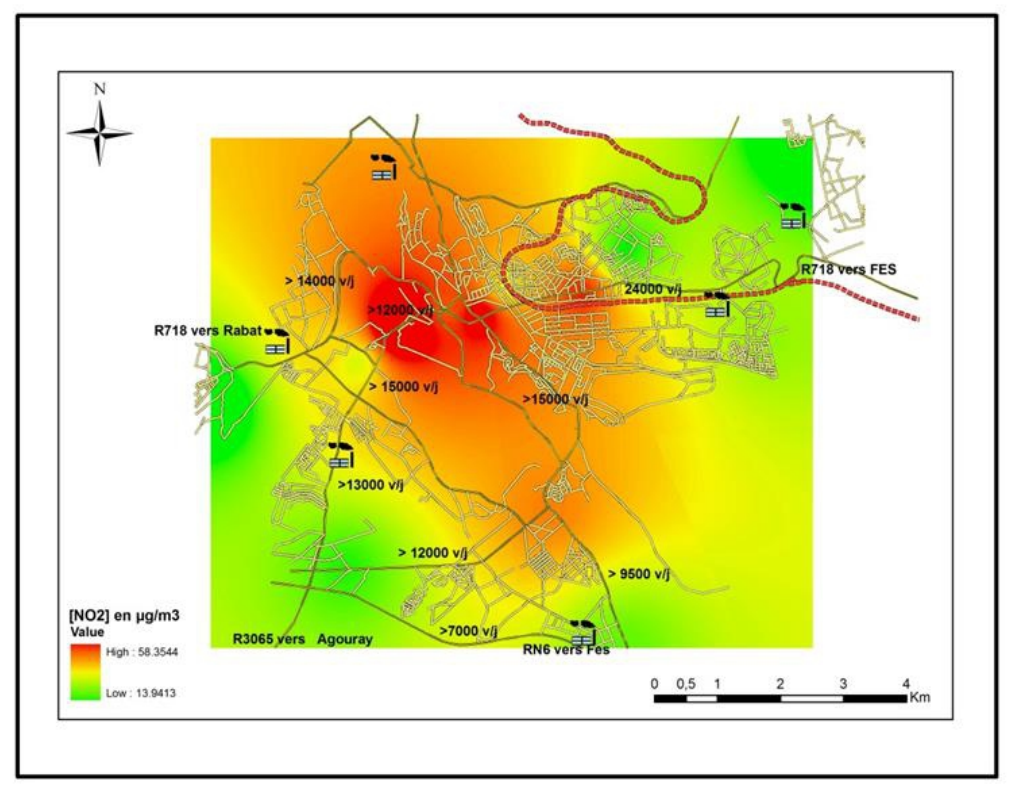

Fig.6(a). Les variations spatiales des teneurs de $\mathrm{NO}_{2}$ au niveau de la ville de Meknès (résultats de la campagne de mesure hivernale) 


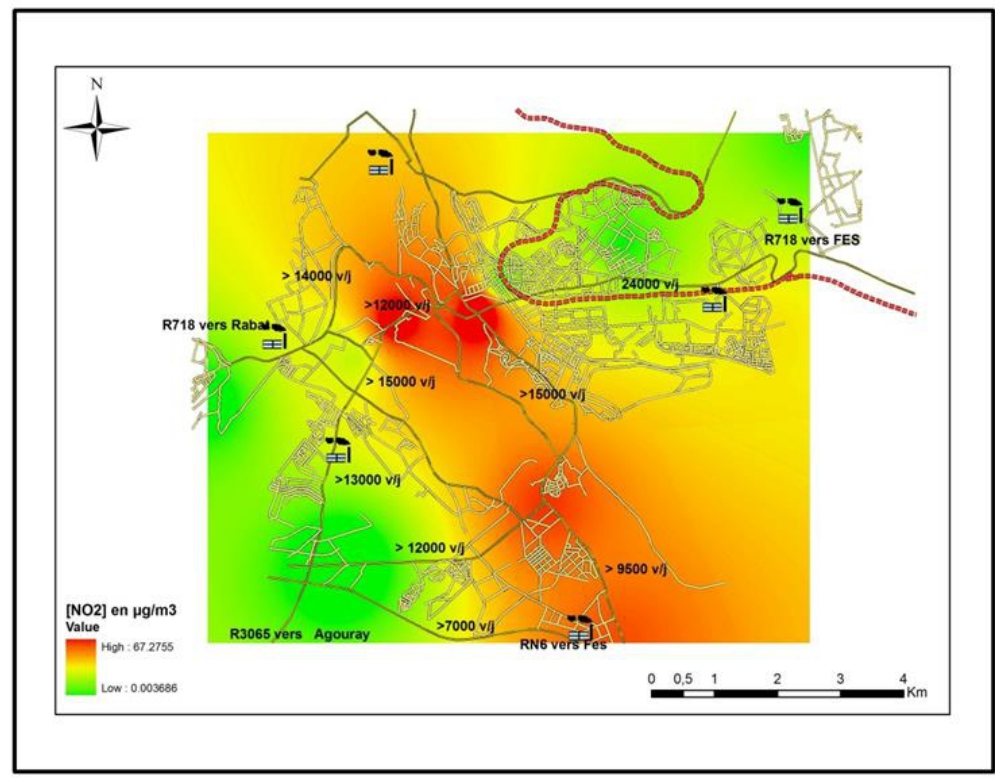

Fig.6(b). Les variations spatiales des teneurs de $\mathrm{NO}_{2}$ au niveau de la ville de Meknès (résultats de la campagne de mesure estivale).

Etude des concentrations de $\mathrm{C}_{6} \mathrm{H}_{6}$

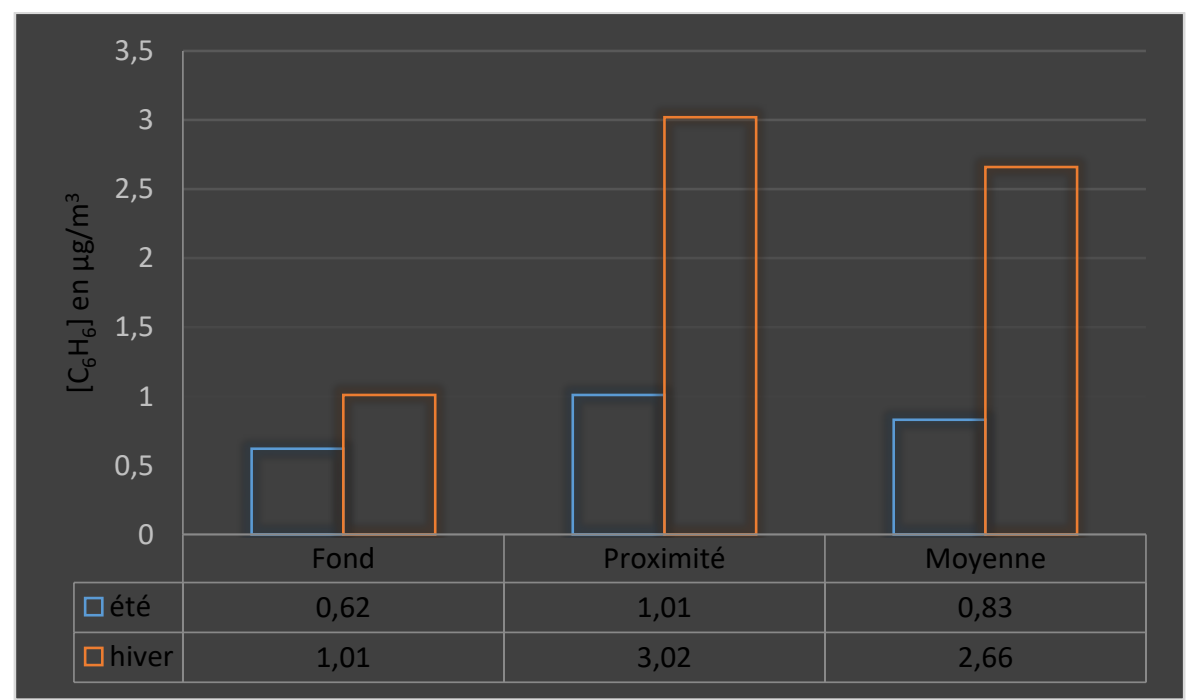

Figure 7. Concentrations moyennes de $\mathrm{C}_{6} \mathrm{H}_{6}$ au niveau de la ville de Meknès durant la période d'étude.

La moyenne des concentrations relevées dans les sites de proximité automobiles $\left(2,04 \mu \mathrm{g} / \mathrm{m}^{3}\right)$ est plus élevée que celle des sites de fond $(1,40$ $\mu \mathrm{g} / \mathrm{m}^{3}$ ) (fig.7). 
La concentration moyenne de $\mathrm{C}_{6} \mathrm{H}_{6}$ mesurée durant la campagne hivernale $\left(2,66 \mu \mathrm{g} / \mathrm{m}^{3}\right)$ est trois fois plus élevée que celle notée en été $(0,83$ $\mu \mathrm{g} / \mathrm{m}^{3}$ ) (fig.7).

En hiver, la concentration moyenne de $\mathrm{C}_{6} \mathrm{H}_{6}$ dans les sites de proximité automobiles est égale à $3,02 \mu \mathrm{g} / \mathrm{m}^{3}$ alors que celle des sites de fond est de $2,17 \mu \mathrm{g} / \mathrm{m}^{3}$ (fig.7).

En été, la concentration moyenne de $\mathrm{C}_{6} \mathrm{H}_{6}$ était de $1,01 \mu \mathrm{g} / \mathrm{m}^{3}$ au niveau des sites de proximité automobiles contre $0,62 \mu \mathrm{g} / \mathrm{m}^{3}$ dans les sites de fond (fig.7).

La chute des teneurs atmosphériques de $\mathrm{C}_{6} \mathrm{H}_{6}$ par rapport à la campagne de mesure hivernale est probablement attribuée à la réduction du nombre de véhicules fréquentant les principaux axes routiers durant les vacances (Meybeck et al., 2000).

$10 \mu \mathrm{g} / \mathrm{m}^{3}$ est le seuil marocain, respecté sur l'ensemble des sites (fig.8). Au niveau de 4 sites parmi 14, il y a un dépassement de l'objectif de qualité fixé par l'Union Européenne $\left(2 \mu \mathrm{g} / \mathrm{m}^{3}\right)$. Tous les sites de fond présentent des teneurs de $\mathrm{C}_{6} \mathrm{H}_{6}$ inférieures à $2 \mu \mathrm{g} / \mathrm{m}^{3}$, valeur limite tolérée en Europe (Directive 2008/50/CE).

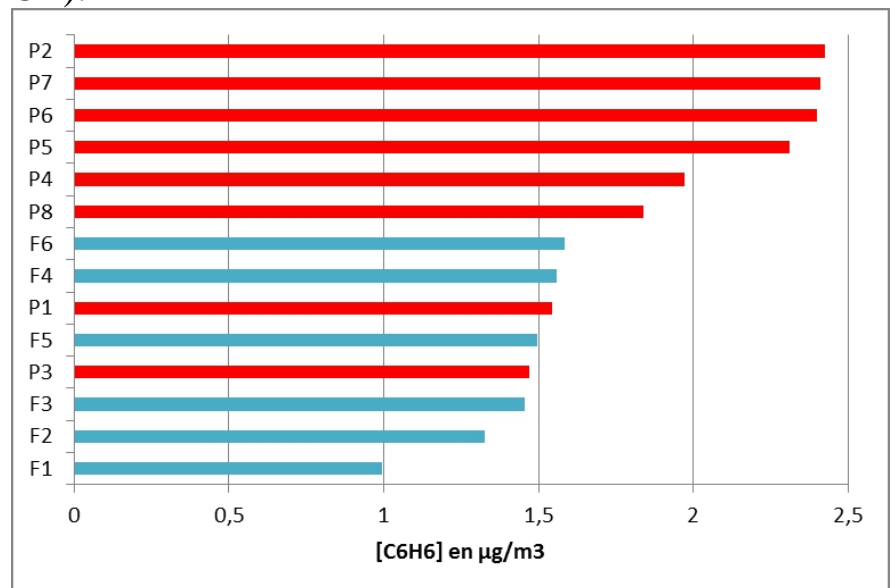

Fig.8. Distribution des sites par concentration de $\mathrm{C}_{6} \mathrm{H}_{6}$ (résultats des campagnes de mesure)

\section{Cartographie des teneurs de $\mathrm{C}_{6} \mathrm{H}_{6}$}

Les plus fortes teneurs de $\mathrm{C}_{6} \mathrm{H}_{6}$ sont enregistrées dans les sites de proximité automobiles, localisés au niveau des axes routiers les plus empruntés du centre-ville (fig.9(a) et 9(b)). 


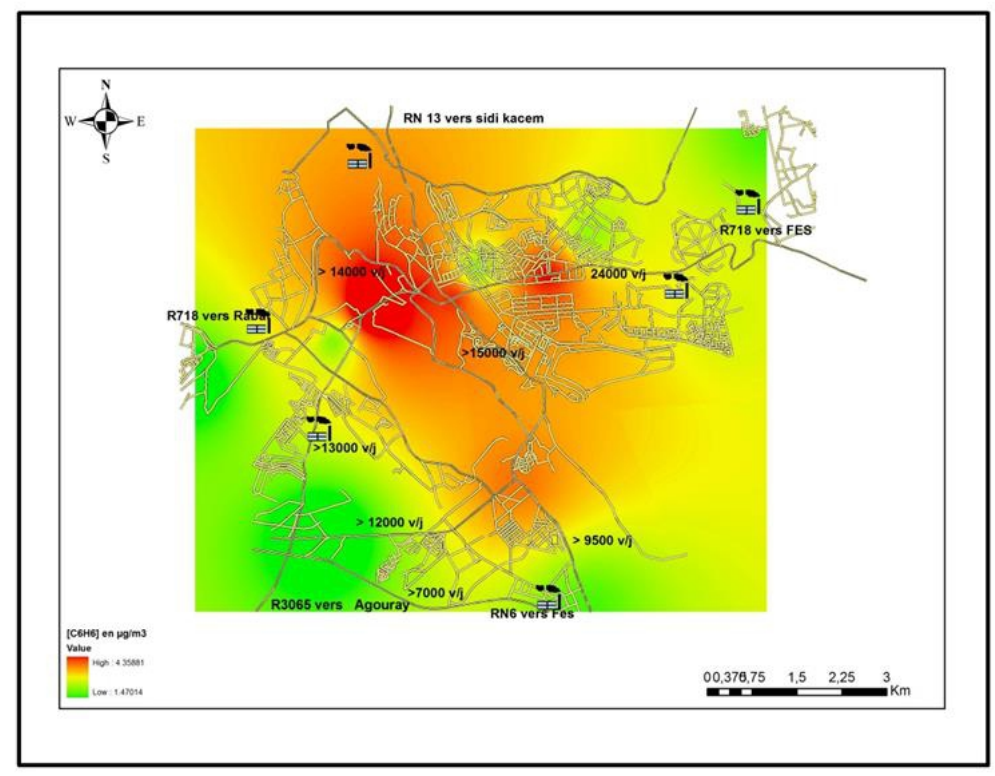

Fig.9(a). Les variations spatiales des teneurs de $\mathrm{C}_{6} \mathrm{H}_{6}$ dans la ville de Meknès (résultats de la campagne de mesure hivernale)

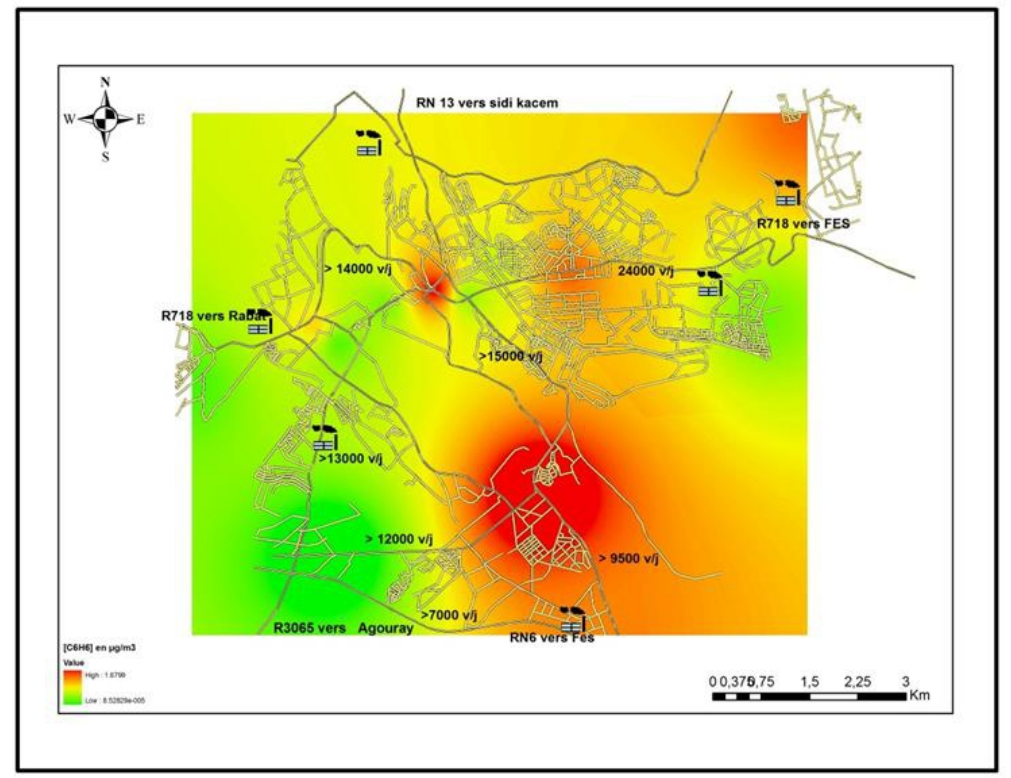

Fig.9(b). Les variations spatiales des teneurs de $\mathrm{C}_{6} \mathrm{H}_{6}$ dans la ville de Meknès (résultats de la campagne de mesure estivale).

Les sites où les teneurs les plus élevées de $\mathrm{C}_{6} \mathrm{H}_{6}$ ont été notées sont : P2 qui représente un point d'intersection de l'avenue Bir Anzarane et le 
boulevard zitoune fréquenté par plus de $20000 \mathrm{veh} / \mathrm{j}\left(2,42 \mu \mathrm{g} / \mathrm{m}^{3}\right)$, P7 localisé au niveau de la rue Dar Smen (>12000 veh/j) dont la structure (rue canyon) s'oppose à la dispersion de $\mathrm{C}_{6} \mathrm{H}_{6}$ et favorise par contre son accumulation $(2,41$ $\left.\mu \mathrm{g} / \mathrm{m}^{3}\right)$ et le site P6 qui lie l'ancienne Medina à la ville nouvelle $\left(2,40 \mu \mathrm{g} / \mathrm{m}^{3}\right)$.

Comme pour le $\mathrm{NO}_{2}$, les sites de mesure situés non loin des zones industrielles présentent de faibles teneurs de $\mathrm{C}_{6} \mathrm{H}_{6}$ ( $\mathrm{P} 1$ et F6).

\section{Conclusion}

La présente étude s'articule sur la surveillance et l'évaluation de la qualité de l'air dans la ville de Meknès, en se basant sur deux traceurs de la pollution de proximité automobiles $\mathrm{NO}_{2}$ et $\mathrm{C}_{6} \mathrm{H}_{6}$ émanant du trafic routier. En absence d'une station télémétrique et d'un laboratoire mobile au sein de la ville, le recours à l'utilisation des tubes à diffusion passive, qui sont analysés en différé au laboratoire, était la solution la plus plausible. Le faible coût, la facilité de la mise en œuvre de la technique et la possibilité de couvrir une large zone géographique avec un grand nombre de préleveurs sont autant d'arguments qui plaident en faveur de l'adoption de cette approche.

le couplage des résultats des campagnes de mesures et des sessions de comptage sous SIG a permis de déterminer les zones les plus affectées par la pollution automobiles et de réaliser ainsi une cartographie à haute échelle de résolution spatiale des polluants prospectés.

\section{Reférences:}

1. Abdouh M., El Atrouz A., Mechkouri A., (2004). Profil environnemental de Meknès, $94 \mathrm{p}$.

2. Ait Bouh H., Benyaich F., Bounakhla M., Noack Y., Tahri M., Zahry F., (2013).Variations Saisonnières des particules atmosphériques et ses composants chimiques dans la Ville de Meknès- Maroc, J. Mater. Environ. Sci. 4 (1), 49-62.

3. Atkins D.H.F., (1990). A Passive Diffusion Tube Sampler for the Measurement of Atmospheric $\mathrm{NO}_{2}$. A New Approach. In: Restelli G., Angeletti G. (eds) Physico-Chemical Behaviour of Atmospheric Pollutants. Springer, Dordrecht.

4. Boularab I., El Ghazi I., Mouhaddach O., Marie-Paule Kestemont MP., and Samir El Jaafari S., (2015). Analyse Spatiale de la Pollution Particulaire au niveau de la Ville de Meknès (Maroc). International Journal of Innovation and Applied Studies, 13(4), 781-788.

5. Directive 2008/50/CE du Parlement européen et du Conseil du 21 mai 2008 concernant la qualité de l'air ambiant et un air pur pour l'Europe.

6. Freeman B., Gharabaghi B., Thé J., Munshed M., Faisal S., Meshal A \& Al Aseed A., (2017). Mapping air quality zones for coastal urban 
centers. Journal of the Air \& Waste Management Association, 67(5), 565-581.

7. Hůnová I., (2017).Measurements of ground-level ozone in Czech mountain forests: what we have learnt from using diffusive samplers. European Journal of Environmental Sciences, 7(2), 125-129.

8. Ibrahim El Ghazi \& Samir El Jaafari., (2015). Relation santéenvironnement et qualité de l'air : Cas de la ville de Meknès (Maroc). Editions Universitaires Européennes, 116 p.

9. ISO 16017-2:2003.Air intérieur, air ambiant et air des lieux de travail -- Échantillonnage et analyse des composés organiques volatils par tube à adsorption/désorption thermique/chromatographie en phase gazeuse sur capillaire -- Partie 2: Échantillonnage par diffusion.

10. Meybeck M., Della Massa J-P., Simon V., Grasset E et Torres L., (2000). Etude de la distribution atmosphérique de composés organiques volatils aromatiques : benzène, toluène, xylènes (BTX) et du dioxyde d'azote sur l'agglomération toulousaine. Pollution atmosphérique, 168,569-582.

11. Mine Evci Y., Esen F., Taşdemir Y., (2016). Monitoring of Long-Term Outdoor Concentrations of PAHs with Passive Air Samplers and Comparison with Meteorological Data. Archives of Environmental Contamination and Toxicology, 71(2), 246-256.

12. Monographie régionale de l'environnement(MRE), région de Meknès Tafilalet., (2001), $64 \mathrm{p}$.

13. NBN EN 16339. Air ambiant - Méthode pour la détermination de la concentration du dioxyde d'azote au moyen d'échantillonneurs par diffusion.

14. OMS., (2018). Neuf personnes sur 10 respirent un air pollué dans le monde, communiqué de presse de l'Organisation Mondiale de la Santé, Genève.

15. Plaisance H., Piechocki-Minguy A., Garcia-Fouque S. \& Galloo J. C., (2004). Influence of meteorological factors on the $\mathrm{NO}_{2}$ measurements by passive diffusion tube. Atmospheric Environment, 38(4), 573-580.

16. Sai M., Kestemont M-P., El Jaafari S., (2014). L'implémentation d'un système de management environnemental: Cas de la commune urbaine de Meknès (Maroc). Editions Universitaires Européennes, 196 p.

17. Tahir M., Zulfiqar A., Nausheen N., Sidra S., Zaheer N \& Ian C., (2015). Measurement of $\mathrm{NO}_{2}$ indoor and outdoor concentrations in selected public schools of Lahore using passive sampler. Journal of Animal and Plant Sciences, 25, 681-686.

18. Zabiegała B., Partyka M\& Namieśnik J., (2007). Employment of passive sampling in monitoring indoor air quality in selected 
residences in a Tri-city area in Poland. Toxicological \& Environmental Chemistry, 87(4), 529-541. 